\title{
Modular Enantioselective Synthesis of an Advanced Pentahydroxy Intermediate of Antimalarial Bastimolide A and of Fluorinated and Chlorinated Analogues
}

\author{
Adrien Quintard,*i) Céline Sperandio, and Jean Rodriguez \\ Aix Marseille Univ, CNRS, Centrale Marseille, iSm2, Marseille, France
}

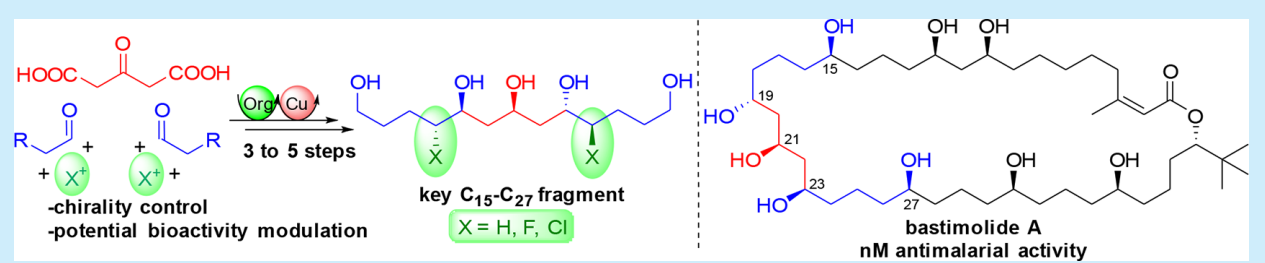

ABSTRACT: A short enantioselective catalytic synthesis of the key C15-C27 fragment of bastimolide A, a natural product showing promising antimalarial bioactivity, is disclosed. The strategic insertion of halogen atoms such as fluorine and chlorine by enantioselective organocatalytic halogenations allowed an excellent stereochemical control for the formation of complex acyclic fragments bearing up to four stereogenic centers. Furthermore, besides the formation of the 1,5,7,9,13-pentahydroxy fragment of the natural product, this strategy opens the route to the modulation of the bioactivity by halogenohydrins.

F or years, the rapid construction of bioactive natural - products has been a source of inspiration pushing synthetic chemists to invent more efficient methodologies facilitating access to key bioactive frameworks. In addition, the proposed strategies also provided access to purely synthetic or simplified analogues with improved or original biological profiles, opening new perspectives for drug development. ${ }^{1}$ The presence or strategic insertion into bioactive molecules of halogen atoms such as chlorine ${ }^{2}$ or fluorine ${ }^{3}$ strongly impacts the observed activity. For example, the group of Carreira recently reported the synthesis of fluoro-, chloro-, and bromodanicalipin A where three different halogenated natural products displayed totally different biological profiles. ${ }^{4}$

The polyketide motif is a crucial molecular arrangement present in numerous natural molecules and drugs. ${ }^{5}$ The complexity of polyketides inspired chemists for decades, but strategies to access these structures still often rely on lengthy stepwise sequences. ${ }^{6}$ Bastimolide A is a natural polyhydroxy macrolide recently isolated from a cyanobacteria whose structure has been firmly established by X-ray analysis (Scheme 1a). ${ }^{7}$ Bastimolide $\mathrm{A}$ is remarkable because it shows an excellent $\mathrm{nM}$ antimalarial bioactivity notably against resistant human parasites. ${ }^{8}$ Given the increasing prevalence of malarial resistance, the apparently new mode of action of bastimolide A is highly promising but requires the identification of efficient synthetic routes amenable to potential structural modulation. However, the core backbone of this natural product encompasses a central acyclic 1,5,7,9,13-pentahydroxy motif (C15-C27 fragment), especially difficult to prepare rapidly and stereoselectively. The enantiomeric 1,5,7,9,13-polyol arrangement is also encountered in caylobolide natural
Scheme 1. Bastimolide A/Caylobolide A and Proposed Strategy

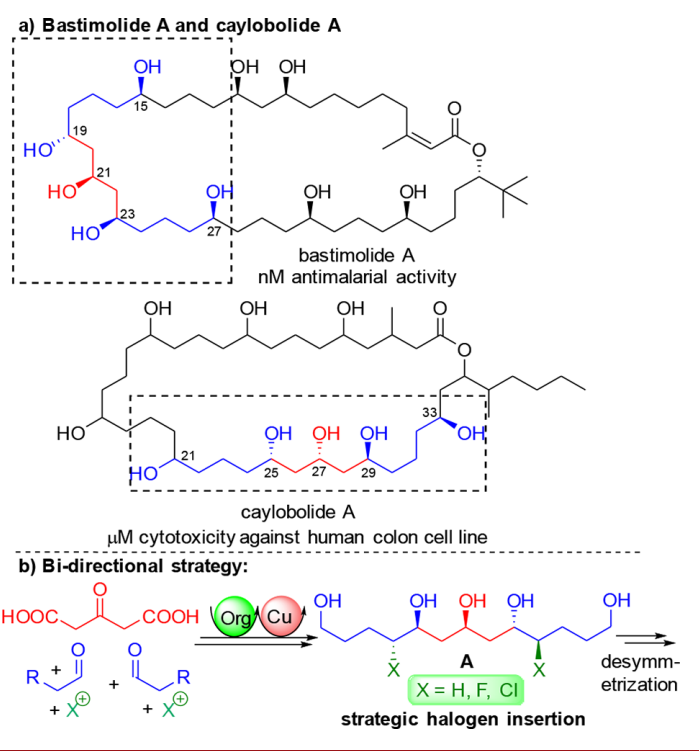

products, another class of macrolides showing interesting cytotoxicity. ${ }^{9}$ While no progress toward bastimolide A 
synthesis has been reported so far, the preparation of the related linear arrangements of caylobolide $\mathrm{A}$ was the topic of two recent studies mostly based on step by step construction of the different alcohols. ${ }^{10}$ In this broad context of polyol synthesis, a pending challenge is to discover eco-compatible methodologies elaborating rapidly acyclic structures while controlling the formation of multiple stereogenic centers with maximum efficiency. Furthermore, in order to improve the bioactivity, such approaches should be modular enough to accommodate the selective introduction of key halogen atoms such as fluorine or chlorine.

Our hypothesis was that the central part of bastimolide A should be easily accessible through the $\mathrm{C}_{2}$-symmetric 1,5,7,9,13-pentahydroxy framework $\mathbf{A}$, from which a final desymmetrization should reveal the three required stereogenic centers of an advanced synthetic intermediate (Scheme 1b). Alcohols A incorporating all the appropriate functions with the right stereochemistry should be readily prepared from simple building blocks with acetonedicarboxylic acid $\mathbf{1}$, aldehydes, and a source of halogen. ${ }^{11}$ This multicomponent assembly should be possible through a multicatalyzed bidirectional didecarboxylative bis-halo-aldolization. Selective halogen insertion would have two main roles in our strategy. It should first control the stereochemistry of the carbon chain by the key diastereoselective aldolization. ${ }^{12}$ In addition, it should provide rapid access to halogenated analogues of our target compound where fluorine or chlorine could modify important properties such as polyol acidity, overall solubility, or conformation. In addition, from the chlorinated aldol adducts, appropriate dehalogenation should rapidly give access to the expected authentic aliphatic fragment as exemplified by the group of Britton. ${ }^{12}$

The primary concern of the study was the compatibility of halogen insertion with the rapid introduction of the hydroxycontaining lateral chain. In order to minimize protecting group manipulation, our initial attempts focused on the fluorinated series with commercially available aldehydes bearing a functionalizable side chain precursor of the terminal hydroxyl function (Scheme 2). In a first approach, Cat.1 was selected for the enantioselective organocatalytic electrophilic fluorination of 4-pentenal (3) with NFSI $2 .{ }^{13}$ Subsequent copper-

Scheme 2. Initial Attempts Using Hydroboration Strategy or Glutaraldehyde Fluorination

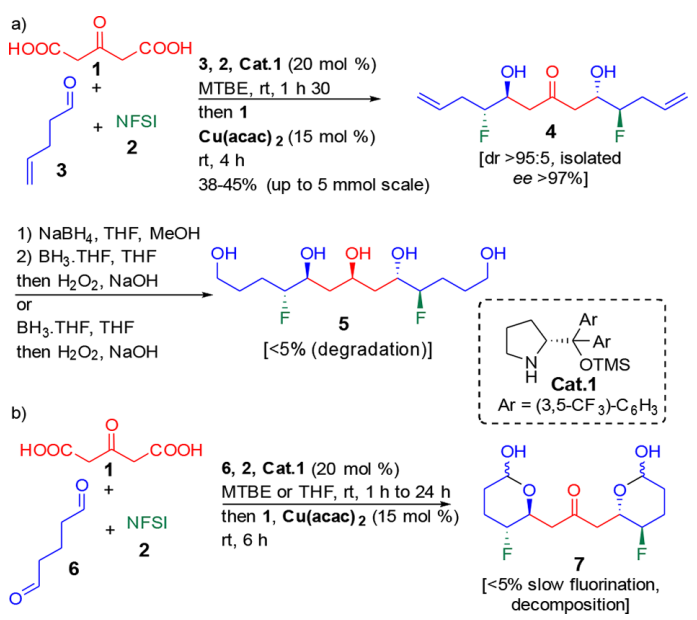

catalyzed diastereoselective bisaldolization with keto-diacid 1 provided the key keto-fluorhydrin $\mathbf{4}$ with excellent stereoselectivity (Scheme 2a). ${ }^{11}$ As a result of the dimerization of the intermediate enantioenriched halogenated aldehyde, as in all these bidirectional reactions, a chirality amplification is observed. At the expense of the yield, this results in the isolation of the keto-diol $\mathbf{4}$ in an excellent enantioselectivity (ee $>97 \%$ ) since undesired minor diastereomers are mostly removed by silica gel chromatography. However, the next required ketone reduction/alkene hydroboration sequence turned to be unproductive, resulting either in decomposition or in the impossibility to recover $\mathbf{5}$ from the aqueous phase. ${ }^{14}$ Alternatively, glutaraldehyde used as a potential precursor of keto-bis-hemiacetal 7 proved to be poorly reactive toward the halogenation-aldolization sequence leaving room mostly to decomposition (Scheme $2 b$ ).

These preliminary results indicated that functionalizing the lateral chain represented a considerable challenge. This difficulty prompted us to turn our attention to the use of a protected alcohol strategy (Scheme 3). Gratifyingly, following

Scheme 3. Fluorinated Analogue Synthetic Strategy

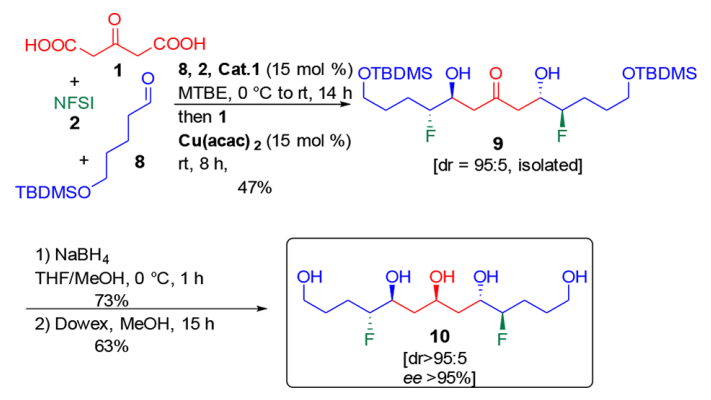

the same approach, aldehyde 8 was fluorinated by Cat.1 with subsequent copper-catalyzed diastereoselective bisaldolization to provide keto-fluorohydrin 9 as a major isolated diastereomer. A sequence of ketone reduction followed by acidic resin promoted removal of the TBDMS group provided the fluorinated bastimolide A analogue fragment 10 with excellent stereoselectivity ( $>95: 5 \mathrm{dr}$ and $>95 \%$ ee). ${ }^{15}$ Noteworthy, when deprotecting the alcohol using classical TBAF conditions, the high water solubility of $\mathbf{1 0}$ prevented its isolation from the otherwise clean reaction mixture, a drawback not encountered with the use of acidic resins.

With this proof of concept for the straightforward synthesis of the key bastimolide fragment in the fluorinated series (only three steps), we next turned our attention to the development of an original bidirectional chlorination/aldolization sequence. The extension of bidirectional aldolization strategies to the chlorinated series should open wide perspectives by providing innovative routes to polychlorinated polyols, but also to access stereoselectively saturated versions which is an ongoing challenge in the field of bidirectional aldolization. ${ }^{16}$

For this purpose, while numerous methods have been developed for the enantioselective $\alpha$-chlorination of aldehydes, we chose simple $\mathrm{N}$-chlorosuccinimide as the chlorinating reagent and L-prolinamide as a cheap organocatalyst. ${ }^{17}$ Even if the widely available prolinamide usually does not provide optimal enantiocontrol in aldehyde chlorination (typically $80-$ $85 \%$ ee), this limitation should be compensated by the 
dimerization of the aldehyde, amplifying the overall enantiopurity of the product.

Unfortunately, first attempts carried out by performing the reaction in one-pot only led to the partial recovery of the intermediate $\alpha$-monochlorinated aldehyde. We hypothesized that this lack of reactivity arose from the inhibition of the copper complex by one of the constituents of the enantioselective chlorination, notably prolinamide or dichloromethane. ${ }^{16 \mathrm{~d}}$ Fortunately, the intermediate $\alpha$-monochlorinated aldehyde was stable enough to allow a rapid removal of most components and solvent prior to aldolization in MTBE restoring the excellent reactivity of the copper catalyst (Scheme 4). Following this two-pot procedure, the chlorinated

Scheme 4. Chlorinated and Natural Polyol Fragment Synthesis

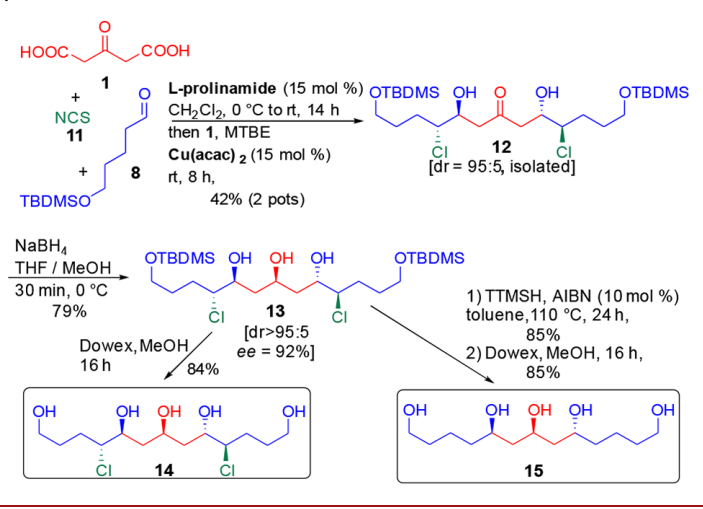

keto-diol 12 possessing four acyclic stereogenic centers could be formed in $42 \%$ overall yield and 95:5 $\mathrm{dr}^{18} \mathrm{NaBH}_{4}$ ketone reduction afforded the crucial triol $\mathbf{1 3}$ with excellent stereoselectivity and $79 \%$ yield. The starting point for the modulation of the synthesis is the silyl group removal from 13 leading to the fully deprotected chlorinated analogue fragment $\mathbf{1 4}$. Alternatively, from 13, radical dechlorination followed by alcohol deprotection gave the 1,5,7,9,13-pentahydroxy motif 15 corresponding to the $\mathrm{C} 15-\mathrm{C} 27$ fragment of the natural product.

In order to highlight the utility of the bidirectional approach, 15 was further elaborated toward a more synthetically relevant advanced intermediate (Scheme 5). Desymmetrization of the C2-symmetrical polyol took place by forming the thermodynamic syn acetal 16. ${ }^{19}$ In this desymmetrized selectively protected polyol now possessing four stereogenic centers, the two terminal primary alcohols can potentially be differentiated by oxidation. The proof of concept of this reactivity was

Scheme 5. Proof of Concept of Desymmetrization of 15

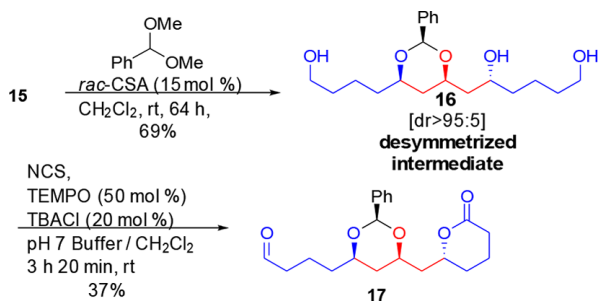

demonstrated using a NCS/TEMPO oxidation mix. This triggers the direct formation of the monoaldehyde 17 from an in situ oxidative lactonization of the intermediate hydroxydialdehyde. Further studies improving this oxidation protocol and using the formed aldehyde in stereoselective allylation should provide a rapid route to bastimolide $A .^{20}$

In conclusion, we have demonstrated that the threecomponent multicatalyzed bidirectional didecarboxylative bishalo-aldolization could provide concise routes to advanced fragments of complex molecules of high biological interest. The enantioselective approach disclosed herein gave access in 5 steps to the $\mathrm{C} 15-\mathrm{C} 27$ fragment of bastimolide A but also to fluorinated as well as chlorinated analogues. The strategic insertion of halogen atoms allowed for the control of the stereoselectivity of the synthetic sequence. In addition, the location of these halogens adjacent to alcohols should modify the acidity of these polyols, opening new perspectives in the understanding and applications of such motifs in drugs. ${ }^{21}$ Finally, this type of multicatalytic approach should find broader applications to construct rapidly other complex molecules.

\section{AUTHOR INFORMATION}

\section{Corresponding Author}

*E-mail: adrien.quintard@univ-amu.fr. ORCID

Adrien Quintard: 0000-0003-0193-6524 Author Contributions

The manuscript was written through contributions of all authors. All authors have given approval to the final version of the manuscript.

Notes

The authors declare no competing financial interest.

\section{ACKNOWLEDGMENTS}

The Centre National de la Recherche Scientifique (CNRS) and the Aix-Marseille Universite are gratefully acknowledged for financial support.

\section{REFERENCES}

(1) See for example: DeChristopher, B. A.; Loy, B. A.; Marsden, M. D.; Schrier, A. J.; Zack, J. A.; Wender, P. A. Nat. Chem. 2012, 4, 705

(2) For reviews: (a) Cabrita, M. T.; Vale, C.; Rauter, A. P. Mar. Drugs 2010, 8, 2301. (b) Gribble, G. W. Mar. Drugs 2015, 13, 4044. (c) Gál, B.; Bucher, C.; Burns, N. Z. Mar. Drugs 2016, 14, 206.

(3) For reviews on F insertion: (a) Purser, S.; Moore, P. R.; Swallow S.; Gouverneur, V. Chem. Soc. Rev. 2008, 37, 320. (b) Wang, J.; Sánchez-Roselló, M.; Aceña, J. L.; del Pozo, C.; Sorochinsky, A. E.; Fustero, S.; Soloshonok, V. A.; Liu, H. Chem. Rev. 2014, 114, 2432.

(4) (a) Bailey, A. M.; Wolfrum, S.; Carreira, E. M. Angew. Chem. Int. Ed. 2016, 55, 639. (b) Fischer, S.; Huwyler, N.; Wolfrum, S.; Carreira, E. M. Angew. Chem., Int. Ed. 2016, 55, 2555. For another example of chlorinated bioisostere synthesis, see: (c) Krautwald, S.; Nilewski, C.; 
Mori, M.; Shiomi, K.; Omura, S.; Carreira, E. M. Angew. Chem. Int. Ed. 2016, 55, 4049 .

(5) (a) Rohr, J. Angew. Chem. Int. Ed. 2000, 39, 2847. (b) Koskinen, A. M. P.; Karisalmi, K. Chem. Soc. Rev. 2005, 34, 677.

(6) (a) Staunton, J.; Weissman, K. J. Nat. Prod. Rep. 2001, 18, 380.

(b) Bode, S. E.; Wolberg, M.; Müller, M. Synthesis 2006, 4, 557.

(c) Schetter, B.; Mahrwald, R. Angew. Chem. Int. Ed. 2006, 45, 7506.

(d) Kumar, P.; Tripathi, D.; Sharma, B. M.; Dwivedi, N. Org. Biomol.

Chem. 2017, 15, 733. For enzymatic approach towards fluorinated polyketide: (e) Walker, M. C.; Thuronyi, B. W.; Charkoudian, L. K.; Lowry, B.; Khosla, C.; Chang, M. C. Y. Science 2013, 341, 1089.

(7) Shao, C.-L.; Linington, R. G.; Balunas, M. J.; Centeno, A.; Boudreau, P.; Zhang, C.; Engene, N.; Spadafora, C.; Mutka, T. S.; Kyle, D. E.; Gerwick, L.; Wang, C.-Y.; Gerwick, W. H. J. Org. Chem. 2015, 80, 7849.

(8) Another family member, Bastimolide B, consisting of the same C15-C27 motif with different macrocycle size, has recently been isolated: Shao, C.-L.; Mou, X.-F.; Cao, F.; Spadafora, C.; Glukhov, E.; Gerwick, L.; Wang, C.-Y.; Gerwick, W. H. J. Nat. Prod. 2018, 81, 211.

(9) (a) MacMillan, J. B.; Molinski, T. F. Org. Lett. 2002, 4, 1535. The $\mathrm{C} 1-\mathrm{C} 21$ stereochemistry of the polyols is unknown. For other family members, see: (b) Salvador, L. A.; Paul, V. J.; Luesch, H. J. Nat. Prod. 2010, 73, 1606.

(10) (a) De Joarder, D.; Jennings, M. P. Tetrahedron Lett. 2011, 52, 5124. (b) Yadav, J. S.; Swapnil, N.; Venkatesh, M.; Prasad, A. R. Tetrahedron Lett. 2014, 55, 1164. For the synthesis of the C1-C24 fragment, see: (c) De Joarder, D.; Jennings, M. P. Tetrahedron Lett. 2013, 54, 5826

(11) (a) Quintard, A.; Rodriguez, J. ACS Catal. 2017, 7, 5513.

(12) See ref 11 and: (a) Britton, R.; Kang, B. Nat. Prod. Rep. 2013 30, 227. (b) Kang, B.; Mowat, J.; Pinter, T.; Britton, R. Org. Lett. 2009, 11, 1717. (c) Borg, T.; Danielsson, J.; Somfai, P. Chem. Commun. 2010, 46, 1281. (d) Halperin, S. D.; Britton, R. Org. Biomol. Chem. 2013, 11, 1702. (e) Bergeron-Brlek, M.; Meanwell, M.; Britton, R. Nat. Commun. 2015, 6, 6903.

(13) Marigo, M.; Fielenbach, D.; Braunton, A.; Kjaersgaard, A.; Jørgensen, K. A. Angew. Chem. Int. Ed. 2005, 44, 3703.

(14) Protection of the triol through the formation of a cyclic acetal prior to hydroboration also did not allow the isolation of the expected product. See Supporting Information for details.

(15) Enantioselectivity was determined using Mosher's ester technique.

(16) (a) Mikami, K.; Matsukawa, S.; Nagashima, M.; Funabashi, H.; Morishima, H. Tetrahedron Lett. 1997, 38, 579. (b) Shimoda, Y.; Kubo, T.; Sugiura, M.; Kotani, S.; Nakajima, M. Angew. Chem. Int. Ed. 2013, 52, 3461. (c) Valero, G.; Ribo, J. M.; Moyano, A. Chem. Eur. J. 2014, 20, 17395. (d) Quintard, A.; Rodriguez, J. Chem. Eur. J. 2015, 21, 14717. (e) Kotani, S.; Kai, K.; Shimoda, Y.; Hu, H.; Gao, S.; Sugiura, M.; Ogasawara, M.; Nakajima, M. Chem. Asian J. 2016, 11, 376. (f) Kotani, S.; Kai, K.; Sugiura, M.; Nakajima, M. Org. Lett. 2017, 19, 3672. (g) Ricucci, A.; Rodriguez, J.; Quintard, A. Eur. J. Org. Chem. 2018, 2018, 3697.

(17) For enantioselective chlorination of aldehydes notably using prolinamide, see: (a) Halland, N.; Braunton, A.; Bachmann, S.; Marigo, M.; Jørgensen, K. A. J. Am. Chem. Soc. 2004, 126, 4790. (b) Brochu, M. P.; Brown, S. P.; Macmillan, D. W. J. Am. Chem. Soc. 2004, 126, 4108. (c) Marquez, C. A.; Fabbretti, F.; Metzger, J. O. Angew. Chem. Int. Ed. 2007, 46, 6915. (d) Kang, B.; Britton, R. Org. Lett. 2007, 9, 5083. (e) Amatore, M.; Beeson, T. D.; Brown, S. P.; MacMillan, D.W. C. Angew. Chem. Int. Ed. 2009, 48, 5121. See also reference $12 b$.

(18) The diastereoselectivity of the reaction could not be determined precisely but is above $80 \%$ of formation of the major diastereomer that can be isolated by silica gel chromatography. See ref 11 .

(19) (a) Wang, Z.; Deschenes, D. J. Am. Chem. Soc. 1992, 114, 1090. (b) Zhang, Y.; Arpin, C. C.; Cullen, A. J.; Mitton-Fry, M. J.; Sammakia, T. J. Org. Chem. 2011, 76, 7641. (c) Shepherd, J. N.; Na,
J.; Myles, D. C. J. Org. Chem. 1997, 62, 4558. (d) Hartmann, E.; Oestreich, M. Org. Lett. 2012, 14, 2406.

(20) Dechert-Schmitt, A-M. R.; Schmitt, D. C.; Gao, X.; Itoh, T.; Krische, M. J. Nat. Prod. Rep. 2014, 31, 504.

(21) Anti relationship in fluorohydrins is believed to increase the $\mathrm{p} K_{\mathrm{a}}$ of the alcohol: Graton, J.; Wang, Z.; Brossard, A.-M.; Goncalves Monteiro, D.; Le Questel, J.-Y.; Linclau, B. Angew. Chem. Int. Ed. 2012, 51, 6176 